\title{
A stable aberrant immunophenotype characterizes nearly all cases of cutaneous T-cell lymphoma in blood and can be used to monitor response to therapy

\author{
LaBaron T Washington ${ }^{1}$, Yang O Huh ${ }^{1}$, Linda C Powers ${ }^{1}$, Madeleine Duvic ${ }^{2}$ \\ and Dan Jones*1
}

\author{
Address: ${ }^{1}$ Department of Hematopathology, The University of Texas MD Anderson Cancer Center, Houston TX, USA and ${ }^{2}$ Department of \\ Dermatology, The University of Texas MD Anderson Cancer Center, Houston TX, USA \\ Email: LaBaron T Washington - labaron.washington@hcahealthcare.com; Yang O Huh - yangohuh@mdanderson.org; \\ Linda C Powers - lpowers@mdanderson.org; Madeleine Duvic - mduvic@mdanderson.org; Dan Jones* - dajones@mdanderson.org \\ * Corresponding author
}

Published: 10 December 2002

BMC Clinical Pathology 2002, 2:5

This article is available from: http://www.biomedcentral.com/1472-6890/2/5

(C) 2002 Washington et al; licensee BioMed Central Ltd. This is an Open Access article: verbatim copying and redistribution of this article are permitted in all media for any purpose, provided this notice is preserved along with the article's original URL.
Received: 13 August 2002

Accepted: 10 December 2002

\begin{abstract}
Background: Abnormal variations in the expression level of some commonly expressed T-cell antigens are a feature of many T-cell malignancies.

Methods: We sought to assess the frequency of such abnormal antigen expression by flow cytometry in peripheral blood (PB) samples from patients with mycosis fungoides (MF) and Sézary syndrome (SS). We correlated presence of morphologically identifiable tumor cells on PB smear with the frequency of abnormalities in the level of expression of CD3, CD4, CD7, CD8 and CD26. We also examined the degree of stability of these abnormal findings in tumor cells over the course of disease. The flow cytometric findings in 100 PB samples from 44 patients, including 38 who had multiple sequential PB samples (2-8 samples each), were assessed.
\end{abstract}

Results: Abnormalities were seen in the expression level of one or more T-cell markers in $4 \mathrm{I}$ cases (93\%) including CD3 in 34\% of patients, CD4 in 54\%, CD26 in $86 \%$ and CD 45 in $40 \%$ (I0 cases tested). In all but 2 cases, the abnormal T-cell immunophenotype remained similar over the course of treatment and correlated with the relative numbers of tumor cells counted on PB smear.

Conclusions: Using a standard T-cell panel, stable phenotypically aberrant T-cell populations representing the tumor are detected in the vast majority of involved PB samples in MF/SS and can be used to monitor response to therapy.

\section{Introduction}

Enumeration of peripheral blood (PB) leukemic cells in mycosis fungoides (MF)/Sézary syndrome (SS) can be difficult by morphological methods. This is due to difficulties in unequivocally distinguishing neoplastic cells from reactive lymphocytes on standard Wright-Giemsa-stained PB smears. Electron microscopic evaluation of blood sam- ples is impractical for routine use. Therefore, most centers have relied on flow cytometric evaluation of such specimens.

Flow cytometric abnormalities in MF/SS that have been correlated with the presence of tumor cells include increased populations of CD4-positive and/or CD7-nega- 
tive T-cells. [1-3] We have recently shown that increases in CD4-positive, CD26-negative T-cell populations are highly associated with the presence of tumor cells in MF/ SS [4].

Another approach to flow cytometric detection of tumor cells has been the identification of phenotypically aberrant populations that express different amounts of cell surface proteins than normal T-cell populations. Such stable abnormal expression levels of pan-T cell markers is a common feature of T cell tumors [5]. We sought to identify the frequency of such abnormalities in MF/SS in PB and to determine whether these immunophenotypic aberrancies are stable over the course of treatment. We report that alterations in expression of multiple T cell markers as detected by flow cytometry are an easily identifiable and stable feature of nearly all cases of MF/SS. Such immunophenotypic aberrancies can be used to quantify tumor cells from a patient over the course of the disease.

\section{Methods}

\section{Patient samples}

We screened all PB smears from patients referred to our hospital between January 1999 and June 2002 who had a diagnosis of cutaneous atypical lymphoid infiltrate, MF, or SS and a had a full PB flow cytometry panel. Cases were considered morphologically positive if they contained at least $1 \%$ tumor cells by manual differential. We also included subsequent involved PB samples from these patients. Most of the PB smears were reviewed by a single hematopathologist (DJ) to ensure relatively uniform criteria in counting of tumor cells. Sézary tumor cells were defined as either those with markedly convoluted nuclear contours or those that were enlarged and had prominent nucleoli. Diagnoses were based on criteria outlined in the updated World Health Organization classification [6]. Patients were diagnosed to have MF if PB involvement occurred subsequent to patch/plaque cutaneous T-cell lymphoma or SS if the presenting features included an erythrodermic rash with $>500 / \mu \mathrm{L}$ PB tumor cells.

All studies were carried out in accordance with the Declaration of Helsinki and followed a laboratory protocol approved by our institutional review board.

\section{Flow cytometry}

We utilized a standard T-cell antigen panel to analyze the 100 PB samples from the 44 patients. 2-, 3- or 4-color flow cytometric analysis was performed on all samples following a standard red cell lysis method using mouse monoclonal antibodies directed against CD45 (clone 2D1, peridin-chlorophyll-a-protein [PerCP]-conjugated, as well as various combinations of CD26 (fluorescien isothiocyanate [FITC]-conjugated), CD3 (SK7, FITC or phycoerythrin [PE]), CD4 (SK3, PE), CD7 (4H9, FITC), CD8
(SK1, FITC or PE), CD19 (SJ25C1, FITC) and CD26 (L272, FITC). In addition, CD2 (S5.2, FITC), CD5 (L17F12, PE), and $\alpha \beta$ T-cell receptor (T10B9, FITC or PE) were done, in a subset of cases. All antibodies were from BD Biosciences, San Jose, CA and analysis was performed using FACScan or FACScaliber cytometers (BD).

For each antibody, negative staining levels were set by comparison to an isotype-matched control. In a subset of 23 cases, cluster analysis using Paint-a-gate ${ }^{\circledast}$ software (BD) was done to correlate the size of the different lymphocyte populations with the phenotypically aberrant T-cell clusters. Aberrant T cell populations were defined as discrete immunophenotypic clusters (representing a tight cluster of $0.5 \%$ or more of analyzed events) exhibiting change in levels of expression of T-cell antigens relative to an internal immunophenotypically normal T-cell population or relative to expected expression of $\mathrm{T}$ cell associated antigens. As a control group for variations in T-cell markers in non-neoplastic T-cell populations, we used the flow cytometry results from 100 patients with MF or cutaneous atypical T-cell infiltrates whose PB smears did not show identifiable tumor cells, and 10 samples from control patients without any evidence of malignancy or chronic disease. The details of these $\mathrm{PB}$ cases were previously summarized [4]. We have previously shown high levels of correlation between the presence of an abnormal T-cell population identified by this flow cytometry panel and the presence of morphologically identifiable tumor cells on examination of the smear [4].

The Kruskal-Wallis test and Spearman's rank correlation test were used to compare the association of phenotypic aberrancy between different markers. Statistical significance was defined as $\alpha=0.05$.

\section{Results}

Immunophenotypic aberrancy of common T-cell markers is present in nearly all cases of MFISS

In 41 of $44 \mathrm{MF} / \mathrm{SS}$ patients (93\%), there was a discrete phenotypically abnormal population(s) identified by flow cytometric analysis that correlated with the presence of morphologically identifiable tumor cells on PB smear. In a group of $100 \mathrm{SS} / \mathrm{MF}$ patients with morphologically uninvolved PB smears, flow cytometric analysis identified a phenotypically abnormal CD4+ T-cell population in only 2 cases. These abnormal populations, detected only by flow cytometry, comprised 1 and $2 \%$ of total white blood cells and were discrete CD4-dim CD26-negative Tcell populations. Rereview of PB smears from these patients, who had typical cutaneous findings of MF, did not identify definitive Sezary cells. The CD4/CD8 ratio was elevated $(>4: 1)$ in $77 \%$ of the involved PB samples, at presentation, and in $74 \%$ of the followup involved $\mathrm{PB}$ samples but in only $13 \%$ of the PB samples from MF/SS 

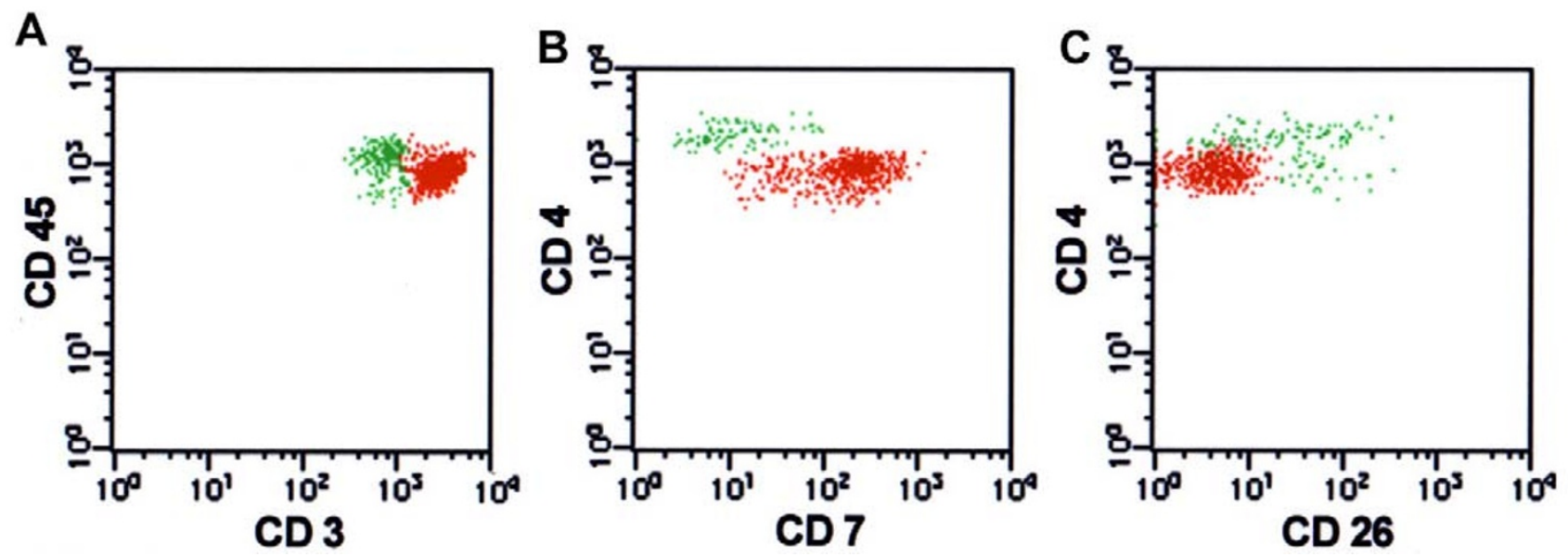

Figure I

Sézary syndrome with multiple immunophenotypic aberrancies in tumor cells. Aberrant events representing neoplastic cells are painted red and normal CD4 and CD8 T-cell populations are painted green. A. Tumor cells are CD3 bright(+)CD45 $\operatorname{dim}(+)$. B. They are also CD4 $\operatorname{dim}(+) C D 7$ bright(+) phenotype and are separable from the normal CD4(+) Tcell population. $C$ Tumor cell population is CD4 $\operatorname{dim}(+)$ CD26(-).

Table I: Overall incidence of aberrancies in common T-cell markers in tumor cells of MF and SS.

\begin{tabular}{ccccccccc}
\hline Aberrancy & CD3 & CD4 & CD5* & CD7 & CD8 & CD26 & CD45* & TCR* \\
\hline \% of cases & $34 \%$ & $54 \%$ & $25 \%$ & $61 \%$ & $5 \%$ & $86 \%$ & $40 \%$ & $31 \%$ \\
cases tested & $15 / 44$ & $24 / 44$ & $5 / 20$ & $27 / 44$ & $2 / 44$ & $38 / 44$ & $4 / 10$ & $6 / 19$ \\
\hline
\end{tabular}

*Markers tested on only a subset of cases.

patients without tumor cells seen on smear. No flow cytometric abnormalities were detected in CD4-positive Tcells from 10 control patients without lymphoproliferative disorders.

The overall incidence of different immunophenotypic aberrancies among the $44 \mathrm{MF} / \mathrm{SS}$ patients is summarized in Table 1. We noted abnormal expression levels of CD3 by flow cytometry in $34 \%$ of patients; tumor cells were dimmer for CD3 than normal T-cell levels in 11 patients, brighter than normal for CD3 in 2 patients (Fig. 1A) and completely negative for CD3 in 2 patients. Similarly, abnormal levels of CD4 expression were seen in $24(54 \%)$ patients ( 21 dimmer, 1 brighter, 2 negative) (Fig. 1B). Coexpression of CD4 and CD8 was seen in tumor cells from 2 patients. In $17 \%$ of patients, tumor cells showed both CD3 and CD4 aberrancies, however the magnitude of these changes were not highly correlated when graded as negative $(0)$, dim (1), normal (2) or bright $(3)$ (R value $=$ $.23)$.
In a subset of cases tested, abnormalities in the dimmer expression level of CD 45 were seen in 4 of $10(40 \%)$ cases (Fig. 1A). T-cell receptor-alpha/beta (TCR- $\alpha / \beta)$ expression levels were determined in 19 cases and was $100 \%$ correlated with CD3 expression, in that $\operatorname{dim}$ TCR- $\alpha / \beta$ expression in 3 cases was associated with $\operatorname{dim}$ CD 3 and TCR- $\alpha / \beta$ was negative in the 2 cases that showed absence of surface CD3. CD5 expression was abnormal in 5 of $20(25 \%)$, of tested cases (dimmer in 4, brighter in 1), by standard analysis. However, the level of CD5 expression in T-cells was typically variable and careful cluster analysis in 5 additional cases identified discrete CD5-dim tumor cell populations.

T-cells in normal PB typically showed a continuous variation in CD7 expression level. In involved MF/SS PB samples, tumor cells were uniformly negative in 17 patients (39\%) and uniformly positive in 10 patients (23\%). In the remaining 17 patients (38\%), CD7 expression in tumor was variable (Fig. 1B) and was often indistinguishable from the range of expression seen in normal T-cells. 

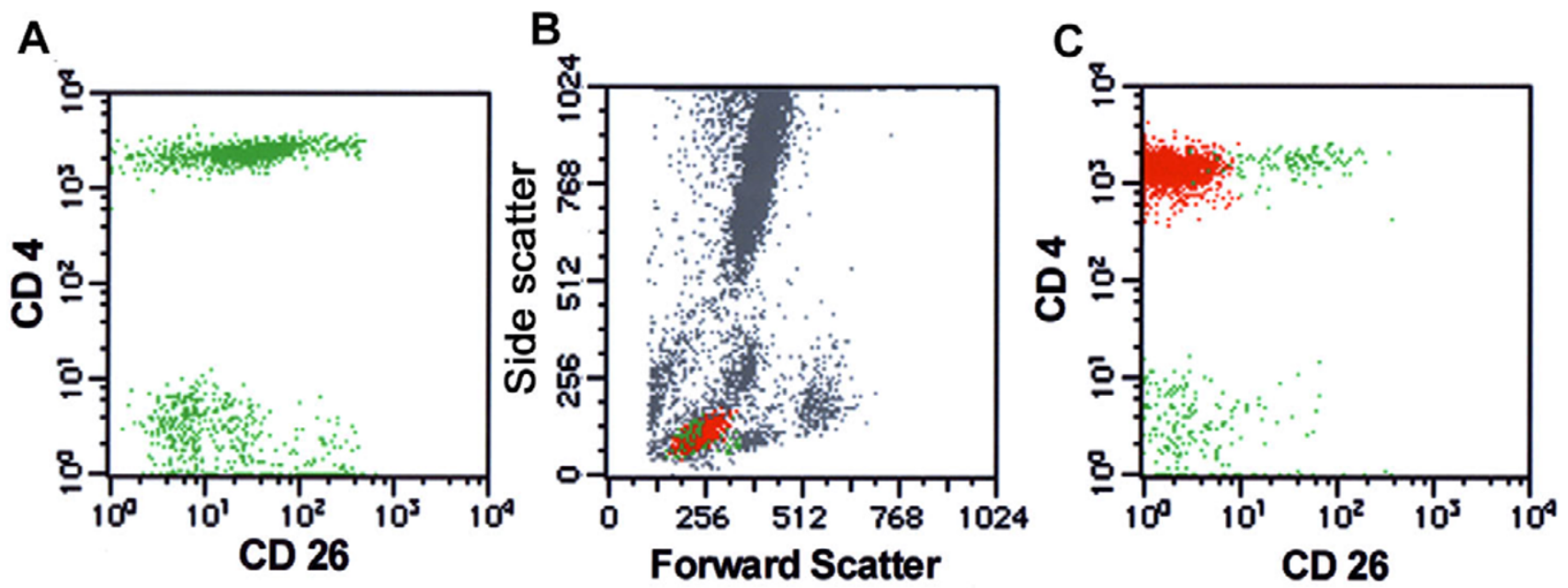

Figure 2

Absence of CD26 expression is useful in isolating neoplastic T-cell populations. A. Non-neoplastic PB shows normal variable expression of CD26 in CD4(+) T-cells. B. The forward/side scatter histogram from a case of MF in PB. Aberrant events representing neoplastic cells are painted red and phenotypically normal CD4 and CD8 T-cell populations are painted green. C. CD4/CD26 histogram shows a discrete uniformly CD26(-) abnormal T-cell population representing tumor cells.

The T-cell activation marker CD26 was uniformly negative in tumor cells from 36 patients $(81 \%)$, uniformly positive in 2 patients and showed variable positivity in 6 . Thus, in 38 of 44 (86\%) patients, CD4/CD26 and CD3/CD26 histograms clearly separated the phenotypically atypical $\mathrm{T}$ cell populations (Figs. 1C and 2).

\section{Multiple phenotypically aberrant T-cell populations in MFISS}

In 5 patients with long standing MF, we noted 2 discrete phenotypically abnormal T-cell populations in PB. In 2 patients, these included a CD3-dim population (Fig. 3A); in 2 other patients there were CD4-dim and CD4-negative populations (Fig. 3B/3C). The remaining patient had two discrete populations detected by CD45 and CD5 staining. In 4 cases, cluster analysis correlated the different phenotypically aberrant populations with the normal-sized and large transformed neoplastic T-cell populations seen on PB smear. This was done by pseudocoloring the abnormal cell cluster in the various antibody panels and correlating the relative cell size of these clusters on the forward/side scatter histograms (Fig. 3A/3C).

\section{Phenotypic aberrancies in T-cells are stable over the course of disease}

In 38 of the patients, we analyzed followup PB samples that had tumor cells identified on smear and a phenotypically abnormal population identified by flow cytometry. We examined from 2 to 8 different samples from these pa- tients over the 3-year study period. In a subset of cases, we used the Paint-a-gate software (on the forward-side scatter and other histograms) to quantify the size of the aberrant (tumor cell) T-cell population in comparison with the other white blood cell types detected. As shown in Table 2, such a technique could be used to document oscillations in tumor cell number that occurred over the course of treatment.

In 36 of 38 patients, phenotypic aberrancies in the levels of CD3, CD 4 and CD26 were stable with similar expression levels detected in all involved PB samples. In contrast, CD7 expression varied in the aberrant tumor cell population in 10 of 38 (26\%) cases. Fig. 4 shows a patient with CD4-dim phenotypic aberrancy in tumor cells that remained constant over the course of disease. In this patient, intervening treatments with Targretin, interferon, and photopheresis had no discernible effect on overall PB tumor cell number. However, initiation of deoxycoformycin (pentostatin) therapy resulted in a decrease in the size of the aberrant CD7-dim tumor cluster (as well as decreases in the population of normal T-cells), consistent with treatment response.

In 2 patients, there were changes in the phenotype of tumor cells noted over the study period. In one case, large cell transformation of tumor cells was associated with partial loss of surface CD3. In another case with CD4-dim 

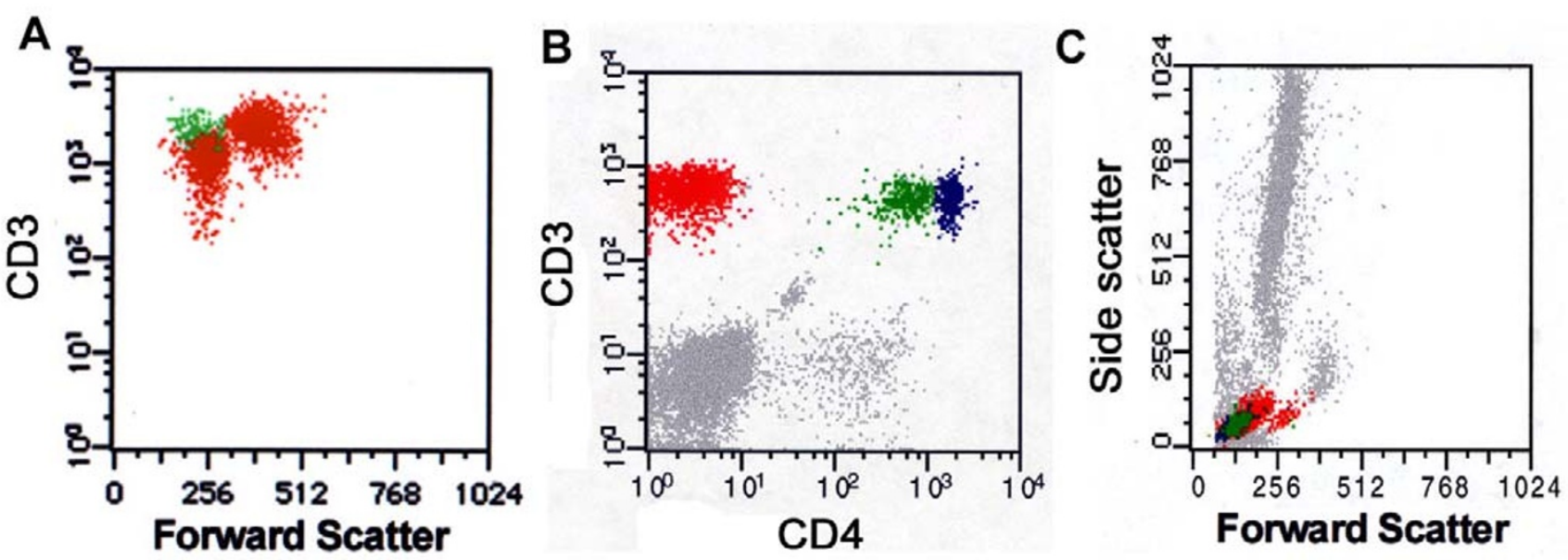

\section{Figure 3}

Mycosis fungoides in peripheral blood with multiple aberrant T-cell populations. A. CD3 versus forward scatter identifies two abnormal populations, one large and one small, that are distinct from normal T cells. Examination of PB smear identified small (cerebriform) and large (transformed) tumor cell populations (not shown). B. Another case of MF in PB shows two atypical T-cell population; one CD4dim(+) (colored green) and another CD4(-) (red) distinct from the normal CD4(+) Tcells (blue). The CD4(-) population was also negative for CD8 (data not shown). C. Forward/side scatter histograms revealed the CD4(-) population predominantly comprised the larger (transformed) tumor lymphocyte population that was noted on PB smear.

Table 2: Variations in the number of neoplastic cells versus normal white cell populations in one MF patient over the course of treatment.

\begin{tabular}{|c|c|c|c|c|c|c|c|}
\hline \multirow[t]{2}{*}{ Date of sample } & \multirow{2}{*}{$\begin{array}{l}\text { White blood cell } \\
\text { count }\left(\times 10^{3} / \mu \mathrm{L}\right)\end{array}$} & \multirow[t]{2}{*}{ Tumor cells* } & \multicolumn{5}{|c|}{ Normal white blood cell populations } \\
\hline & & & CD4 (+) T-cells & CD8 (+) T-cells & NK \& B-cells & grans & monos \\
\hline $7 / 26 / 00$ & 2.8 & $25 \%$ & $6 \%$ & $1 \%$ & $13 \%$ & $42 \%$ & $13 \%$ \\
\hline $9 / 5 / 00$ & 4.2 & $5 \%$ & $2 \%$ & $4 \%$ & $3 \%$ & $85 \%$ & $1 \%$ \\
\hline $9 / 22 / 00$ & 5 & $10 \%$ & $2 \%$ & $3 \%$ & $2 \%$ & $79 \%$ & $4 \%$ \\
\hline $1 \mathrm{I} / \mathrm{I} / 00$ & 5.5 & $16 \%$ & $2 \%$ & $1 \%$ & $4 \%$ & $76 \%$ & $1 \%$ \\
\hline
\end{tabular}

*Tumor cells had a CD3(+)CD4(-)CD8(-) immunophenotype, allowing accurate separation from normal T-cell populations.

and CD4-negative tumor cell populations, effective treatment was associated with selective loss of the CD4-negative population (data not shown).

\section{Discussion}

We examined the flow cytometry findings in involved $\mathrm{PB}$ samples from $44 \mathrm{MF} / \mathrm{SS}$ patients, most of who had multiple sequential involved $\mathrm{PB}$ samples. Using a standard Tcell cytometry panel, we noted abnormal T-cell populations showing one or more antigen aberrancies in 93\% of patients. In $5 \mathrm{MF}$ patients, we noted multiple persistent phenotypically abnormal T-cell populations consistent with clonal heterogeneity. We present the first report on the stability of such abnormal immunophenotypes in the neoplastic T cells of SS/MF, over disease course. Despite varying treatments, immunophenotypic aberrancies noted were maintained over the course of disease in $95 \%$ of cases, excluding common variations in the level of CD7 expression. These findings support the hypothesis that these abnormal T-cell populations represent the neoplastic clone and provide strong support for the use of flow cytometry in routine monitoring of treatment response. 

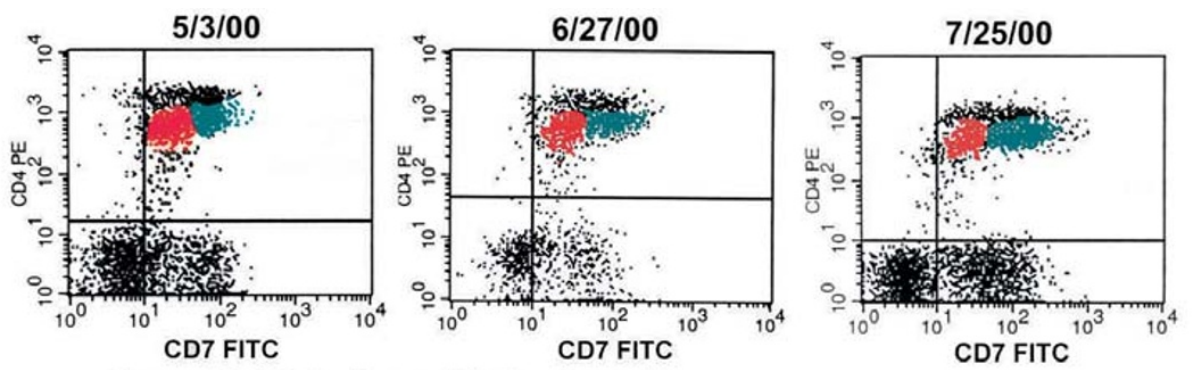

Targretin/Interferon/Photopheresis

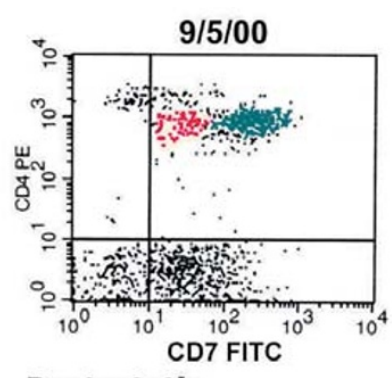

Pentostatin

Figure 4

Stability of abnormal tumor immunophenotype of sequential samples from a SS patients undergoing treatment. There is stable expression of $\operatorname{dim}$ CD4 in the tumor cells, with CD7dim(+) and CD7bright $(+)$ populations painted red and green, respectively. The histogram on the far right shows a decrease in the CD7dim $(+)$ population in response to pentostatin therapy. No overall decreases in tumor cell number in response to the prior Targretin, interferon or photopheresis treatments were noted.

Tumor-associated variation in one or several pan-T cell antigens is a typical feature of neoplastic lymphocytes in many T-cell malignances and has been termed antigen "loss" or "deletion" [7]. Using immunohistochemistry on a large bank of T-cell lymphomas, we have previously noted a frequency of such antigen deletion of $18 \%$ for CD2, $26 \%$ for CD3, 30\% for CD5 and 68\% for CD7 [8]. Flow cytometric analysis can allow a more sensitive assessment of subtle variations in the levels of surface marker expression than immunohistochemistry [9]. In non-neoplastic T-cell populations, we noted relatively narrow range of CD3, CD 4 and CD5 expression with majority of cells clustering within one log intensity. In contrast, the aberrancies noted in expression in T-cell tumor often showed 2-3 logfold intensity differences from the normal populations [5,9-12].

Most routinely assessed T-cell diagnostic markers (e.g. TCR- $\alpha / \beta, \mathrm{CD} 3, \mathrm{CD} 4, \mathrm{CD} 8$ and CD45) are components of the antigen receptor complex and are dynamically regulated during T-cell maturation and activation [13]. In a subset of cases analyzed, we noted that surface CD3 levels frequently correlated with levels of TCR- $\alpha / \beta$, but that CD3 did not always correlate with variations in the levels of surface CD4. We also found variations in MF/SS tumor cells in the expression level of CD45, a phosphatase that regulates TCR activation [14]. The finding of frequent variations in the levels of these TCR-complex proteins in tumors is intriguing and suggests abnormal regulation or formation of the TCR complex in some tumor cells. This could be lead to dysregulated TCR signaling effecting growth regulation.
In previous studies, others and we have noted that MF/SS tumor cells frequently lacked CD26, and that this finding was helpful in identifying the tumor cell population $[4,15]$. In this larger longitudinal series, we confirm these findings and noted that tumor cells showed a consistent pattern of CD26 loss over the course of disease. The stable loss of expression of CD26, a T-cell activation marker, in $\mathrm{MF} / \mathrm{SS}$ suggests that this activation pathway may be nonfunctional in most MF/SS tumors. CD26 is an ectoenzyme, which delivers a costimulatory signal in T-cells; its expression is upregulated on T-cells in response to $\mathrm{mi}$ togenic signals and downregulated through interaction with the insulin-like growth factor II receptor [16]. The consequence of CD26 loss is unclear; CD26 modulates the action of many molecules including the chemotactic activity of some chemokine important for T-cell migration to skin $[17,18]$.

In contrast, we noted that $\mathrm{CD} 7$ expression was frequently modulated in tumor cells over the disease course. Several previous studies have demonstrated that CD7 loss is not a predictable marker for detection of MF/SS cells, as its expression is variable in this tumor type [15,19]. Although there is data to suggest that CD7-negative T-cells may represent a distinct differentiation pathway in normal and neoplastic helper T cells, $[20,21]$ the variable expression of CD7 in MF/SS that we have observed suggests that this marker may be highly modulated by tumor microenvironment and/or treatment.

Identification of a stable tumor-associated immunophenotype in MF/SS allows tumor cell quantification to assess response to therapy. Cluster analysis of abnormal flow cy- 
tometric immunophenotype is more reliable and easier to compare over time than morphologic analysis, and can detect PB involvement in a small number of cases without easily identifiable Sezary cells [12]. Routine flow cytometric profiling is also not as technically demanding as molecular monitoring $[22,23]$ or electron microscopy. Such a separation of tumor from normal T-cells is also important in assessing the effects of therapies on normal leukocytes.

In conclusion, we found that the absence of CD26 and other aberrant surface expression of $\mathrm{T}$ cell-associated markers allowed identification of a discrete, quantifiable population in nearly all MF/SS patients with PB tumor cells. Immunophenotypic aberrancies are maintained consistently over the course of disease and can be used to monitor tumor response to therapy.

\section{Acknowledgments}

We thank Louise Huck and the entire staff of the MDACC Immunology Laboratory for their assistance in this work, which was presented in part at the 2000 Clinical Cytometry Meetings, Austin, TX.

\section{References}

I. Schmidt CA, Przybylski G, Seeger K and Siegert W TCR delta gene rearrangements in acute myeloid leukemia with T-lymphoid antigen expression. Leuk Lymphoma 1995, 20:45-9

2. Kukel S, Reinhold U, Oltermann I and Kreysel HW Progressive increase of CD7-T cells in human blood lymphocytes with ageing. Clin Exp Immunol 1994, 98: I63-8

3. Moll M, Reinhold U, Kukel S, Abken H, Muller R and Oltermann I CD7-negative helper $T$ cells accumulate in inflammatory skin lesions. J Invest Dermatol 1994, 102:328-32

4. Jones D, Dang NH, Duvic M, Washington LT and Huh YO Absence of CD26 expression is a useful marker for diagnosis of T-cell lymphoma in peripheral blood. Am J Clin Pathol 200I, I I 5:885-92

5. Wood KM, Pallesen G, Ralfkiaer E, Warnke R, Gatter KC and Mason DY Heterogeneity of CD3 antigen expression in T-cell lymphoma. Histopathology 1993, 22:31 I-7

6. Jaffe ES, Harris N, Stein H and Vardiman JW Tumours of Hematopoietic and Lymphoid Tissues. Lyon, France: IARC Press 200I,

7. Hastrup N, Ralfkiaer $E$ and Pallesen G Aberrant phenotypes in peripheral T cell lymphomas. J Clin Pathol 1989, 42:398-402

8. Jones D and Dorfman DM Phenotypic characterization of subsets of $T$ cell lymphoma: towards a functional classification of T cell lymphoma. Leuk Lymphoma 200I, 40:449-59

9. Jamal S, Picker LJ, Aquino DB, McKenna RW, Dawson DB and Kroft $\mathrm{SH}$ Immunophenotypic analysis of peripheral T-cell neoplasms. A multiparameter flow cytometric approach. Am J Clin Pathol 200I, I 16:5 I2-26

10. Ginaldi L, Matutes E, Farahat N, De Martinis M, Morilla R and Catovsky D Differential expression of CD3 and CD7 in T-cell malignancies: a quantitative study by flow cytometry. $\mathrm{Br} J$ Haematol 1996, 93:921-7

II. Bogen SA, Pelley D, Charif M, McCusker M, Koh H and Foss F Immunophenotypic identification of Sezary cells in peripheral blood. Am J Clin Pathol 1996, 106:739-48

12. Edelman J and Meyerson H Diminished CD3 Expression Is Useful for Detecting and Enumerating Sézary Cells. Am J Clin Path 2000, I | 4:345-352

13. Vignali DA, Carson RT, Chang B, Mittler RS and Strominger JL The two membrane proximal domains of CD4 interact with the T cell receptor. J Exp Med 1996, I 83:2097-107

14. Volarevic S, Niklinska BB, Burns CM, Yamada H, June $\mathrm{CH}$ and Dumont FJ The CD45 tyrosine phosphatase regulates phosphotyrosine homeostasis and its loss reveals a novel pattern of late $\mathbf{T}$ cell receptor-induced Ca2+ oscillations. J Exp Med 1992, I 76:835-44
15. Bernengo MG, Novelli M, Quaglino P, Lisa F, De Matteis A and Savoia $P$ The relevance of the CD4+CD26- subset in the identification of circulating Sezary cells. Br J Dermatol 200I, I44: I 25-35

16. Ikushima $H$, Munakata $Y$, Ishii $T$, Iwata $S$, Terashima $M$ and Tanaka $H$ Internalization of CD26 by mannose 6-phosphate/insulin-like growth factor II receptor contributes to $T$ cell activation. Proc Natl Acad Sci U S A 2000, 97:8439-44

17. Lambeir AM, Proost P, Durinx C, Bal G, Senten K and Augustyns K Kinetic investigation of chemokine truncation by CD26/ dipeptidyl peptidase IV reveals a striking selectivity within the chemokine family. J Biol Chem 200I, 276:29839-45

18. Ludwig A, Schiemann F, Mentlein R, Lindner B and Brandt E Dipeptidyl peptidase IV (CD26) on T cells cleaves the $C X C$ chemokine CXCLI I (I-TAC) and abolishes the stimulating but not the desensitizing potential of the chemokine. J Leukoc Biol 2002, 72: $|83-9|$

19. Dummer R, Nestle FO, Niederer E, Ludwig E, Laine E and Grundmann $\mathrm{H}$ Genotypic, phenotypic and functional analysis of CD4+CD7+ and CD4+CD7-T lymphocyte subsets in Sezary syndrome. Arch Dermatol Res 1999, 291:307-I I

20. Reinhold U, Liu L, Sesterhenn J and Abken H CD7-negative T cells represent a separate differentiation pathway in a subset of post-thymic helper T cells. Immunology I996, 89:39|-6

21. Rappl G, Muche JM, Abken H, Sterry W, Tilgen W and Ugurel S CD4(+)CD7(-) T cells compose the dominant $T$-cell clone in the peripheral blood of patients with Sezary syndrome. J Am Acad Dermatol 200I, 44:456-6I

22. Vega P, Luthra R, Dunmire V, Medeiros L, Lee S-J and Duvic M Clonal heterogeneity in mycosis fungoides and its relationship to clinical course. Blood 2002, 100:3369-3373

23. Delfau-Larue $M H$, Laroche L, Wechsler J, Lepage E, Lahet $C$ and AssoBonnet $M$ Diagnostic value of dominant $T$-cell clones in peripheral blood in 363 patients presenting consecutively with a clinical suspicion of cutaneous Iymphoma. Blood 2000, 96:2987-92

\section{Pre-publication history}

The pre-publication history for this paper can be accessed here:

http://www.biomedcentral.com/1472-6890/2/5/prepub

Publish with Biomed Central and every scientist can read your work free of charge

"BioMed Central will be the most significant development for disseminating the results of biomedical research in our lifetime. "

Sir Paul Nurse, Cancer Research UK

Your research papers will be:

- available free of charge to the entire biomedical community

- peer reviewed and published immediately upon acceptance

- cited in PubMed and archived on PubMed Central

- yours - you keep the copyright 